\title{
¿Qué piensan las Pacientes sobre la presencia de los Estudiantes de Medicina?
}

\section{What do Patients think about Medical Students?}

\author{
Carlos Santiago Piñel Pérez ${ }^{1,2}$ \\ 1 Servicio de Obstetricia y Ginecología del Hospital Quirónsalud San José \\ 2 Universidad Europea de Madrid; \\ * Correspondencia: carlos.s.pinel@gmail.com ; ORCID ID: https: / / orcid.org/0000-0001-6647-8718 \\ Recibido: 05/04/2021; Aceptado: 25/04/21; Publicado: 05/05/2021
}

Resumen: La interacción con el paciente real es indispensable para la formación del estudiante de Medicina, pero para que la experiencia clínica sea completamente inmersiva, es necesario que exista una adecuada relación paciente-estudiante. En esta sencilla experiencia se muestra cuál es la opinión de los alumnos en cuanto a qué piensan las pacientes de Obstetricia y Ginecología sobre su presencia en el entorno clínico, así como la percepción real de las pacientes. En líneas generales, las pacientes entienden la necesidad de formación de los profesionales médicos del futuro y les gusta formar parte de su aprendizaje. Sin embargo, hay una serie de detalles que hay que tener en cuenta para optimizar la relación paciente-estudiante y lograr una experiencia de rendimiento óptimo para el estudiante, pero que sea segura y confortable para los pacientes.

Palabras clave: percepción del paciente; prácticas clínicas; estudiantes de medicina; seguridad del paciente

Abstract: Interaction with the real patient is essential for medical students training,
but an adequate patient-student relationship is necessary to obtain an inmersive
and complete clinical experience. This article shows the opinion of the students
about what the Obstetrics and Gynecology patients think about their presence in
the clinical environment, as well as the real perception of the patients. In general,
patients understand the need for training of future medical professionals, and they
like to be part of their learning. However, there are several details that must be
taken into account to optimize the patient-student relationship and achieve an
optimal experience for the student, but that is safe and comfortable for the patients.

Keywords: patient perception; clinical practice; medical students; patient safety

Hipócrates, los cirujanos barberos de la Edad Media, los grandes médicos de los que constan registros a lo largo de la historia, las facultades de Medicina del siglo XX... Desde siempre, la enseñanza de la Medicina ha tenido un componente práctico en el que son esenciales dos elementos: el "maestro" y la interacción con el paciente real. La presencia de este último como parte del proceso de aprendizaje del alumno de Medicina es indispensable (1), pues de él se aprende la esencia de la enfermedad, que no es la enfermedad en sí, sino el hombre o la mujer enferma. Cómo la enfermedad afecta a la persona, modifica su vida, sus objetivos, sus sueños y deseos. Su realidad. Y esta transformación de la realidad vital del 
paciente que ocasiona la enfermedad, sólo se aprende del enfermo. También la enseñanza sobre el paciente real permite al alumno ver las entidades más frecuentes, y los síntomas más habituales. Pueden percibir el camino de la práctica clínica de una manera más clara. La Medicina en estado puro: del síntoma al proceso lógico de diagnóstico diferencial y de este al diagnóstico definitivo. Es más didáctico, por lo impactante, ver a un paciente con una apendicitis aguda, que estudiarlo en los libros. Es más evidente ver a una mujer resoplando, con trabajo de parto iniciado, que saber que el parto se inicia cuando se cumplen los criterios de Friedman.

Si el alumno logra una adecuada inmersión en el entorno clínico y, de verdad, interactúa con el paciente, la experiencia de aprendizaje será completa, y el poso de conocimiento, profundo. Porque se olvida menos lo que se ha experimentado en primera persona, que lo que se ha estudiado (2). Pero para esa inmersión, hace falta una confianza que debe venir del tutor clínico (es decir, el médico con el que está en ese momento) y del paciente. Yo he observado cómo mis alumnos, muchas veces, tienen miedo a preguntar, a observar, a interactuar con las pacientes... Y creo que es por inseguridad sobre lo que le parece a la paciente. ¿Qué piensa esta mujer sobre mí? ¿Le parecerá bien que yo le hable? ¿Que yo la observe? ¿Que yo le haga la ecografía? Y esa inseguridad es una barrera hacia la experiencia inmersiva que debería suponer la práctica clínica para ellos.

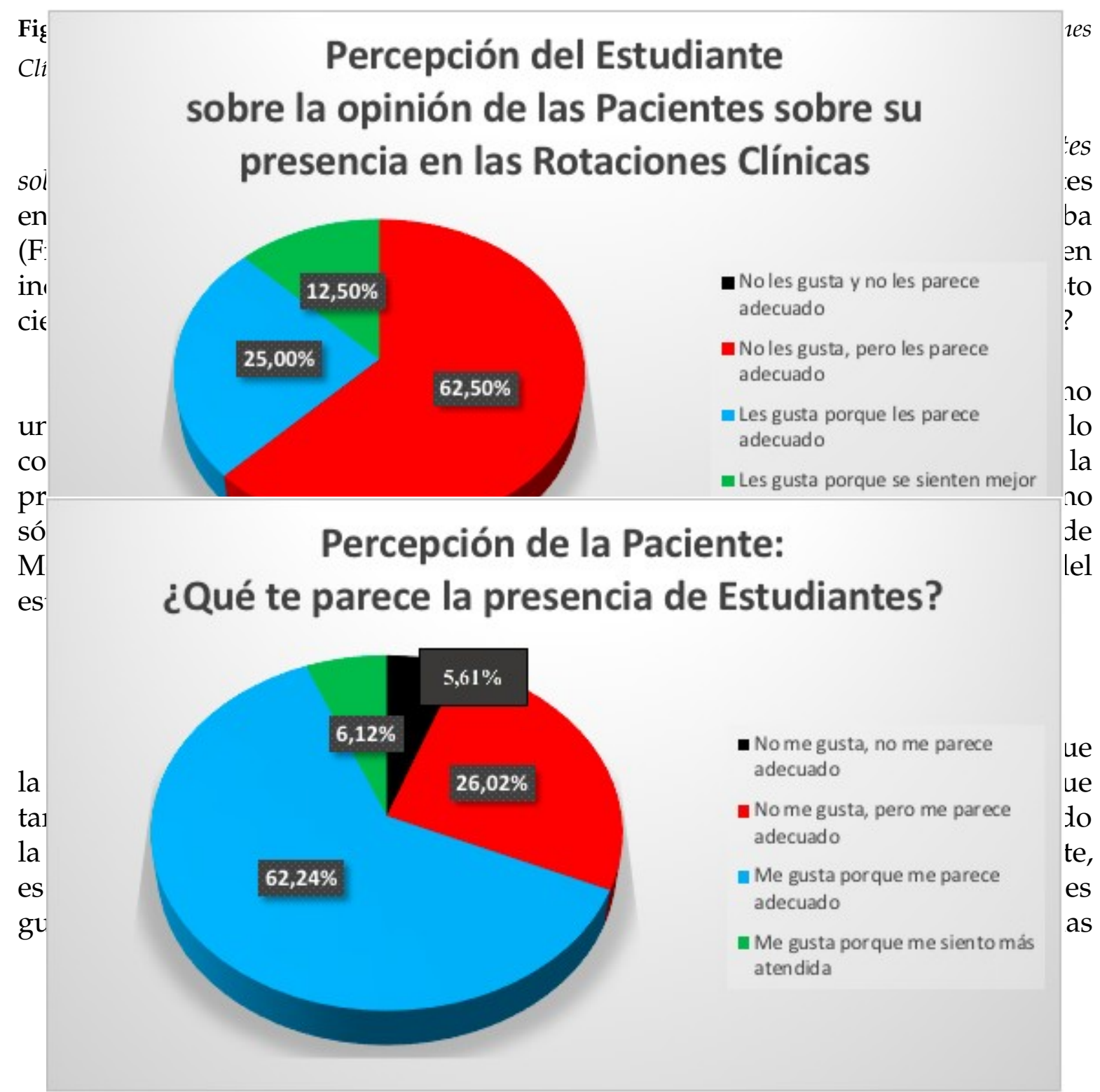


respuestas a la pregunta abierta: ¿Qué os parece la presencia de estudiantes? Y probablemente de estos comentarios se puede extraer qué le gustaría o qué echa en falta la paciente en esa interacción con el estudiante. Y esto es lo que deberíamos aplicar para convertir la experiencia en satisfactoria para la paciente a la par que útil e inmersiva para el estudiante.

Un aspecto común, tanto entre las pacientes a las que les gusta como a las que no la presencia del alumno en el proceso clínico, es el hecho de ser conscientes de la condición de estudiante.

"Me parece que es lo que debe ser, previa presentación, me es igual de cómodo a cuando está el médico solo".

"Me gustaría que el doctor fuera transparente y se lo dijera al paciente. Da más confianza".

"Lo que no me gusta es que no se comente nada y la exploración la haga el estudiante y el ginecólogo".

"Me parece que está bien sólo si se le consulta previamente a la paciente y está de acuerdo".

"Para mí está bien si se presentan y te piden permiso para estar".

"No me parece mal, pero se debe pedir tu consentimiento y nunca se hace".

Obviamente, y no puede ser de otra manera desde un punto de vista ético, la paciente debe saber que ahí hay un estudiante y cuál va a ser su participación.

Por otro lado, una paciente habla de que se debe garantizar que el estudiante posea unas habilidades mínimas antes de dejarles ejecutar procedimientos que pueden verse afectados en su resultado por la falta de experiencia.

"Es algo importante para ellos de cara al futuro, pero hay que estar muy seguros a la hora de dejarles hacer un procedimiento ya que a mí me hicieron mal un punto del parto".

Lógicamente, los estudiantes carecen de experiencia real en la mayoría de los casos. Sin embargo, hoy en día existen magníficos modelos de simulación que deben actuar como paso previo a la interacción con el paciente: pruebas, exploraciones, cirugías. Además, antes de permitir a un estudiante ejecutar un acto médico, debemos estar seguros del rendimiento académico que va a tener esta acción, y decidir si permitirle hacerlo valorando previamente el balance riesgobeneficio (Figura 3). Por ejemplo, una exploración en un abdomen agudo indiscutiblemente va a ser útil para la formación como médico del alumno, ya que va a ser una situación a la que muy probablemente se enfrentará en su futuro profesional. Además, es una actuación que, independientemente de cómo la ejecute, difícilmente va a repercutir en el pronóstico del paciente. Por el contrario, dejar a un estudiante suturar un desgarro en un parto no va a tener un impacto importante en su formación como médico general $\mathrm{y}$, sin embargo, si lo realiza erróneamente puede ocasionar una disfunción del suelo pélvico para la paciente. 
Puede haber situaciones intermedias en las que realizar el procedimiento no sea imprescindible para su formación, pero que no ponen en riesgo el pronóstico del paciente, con lo que sí se puede permitir que el estudiante realice, siempre bajo supervisión, dicho procedimiento. Un ejemplo de esta situación sería una ecografía obstétrica de tercer trimestre.
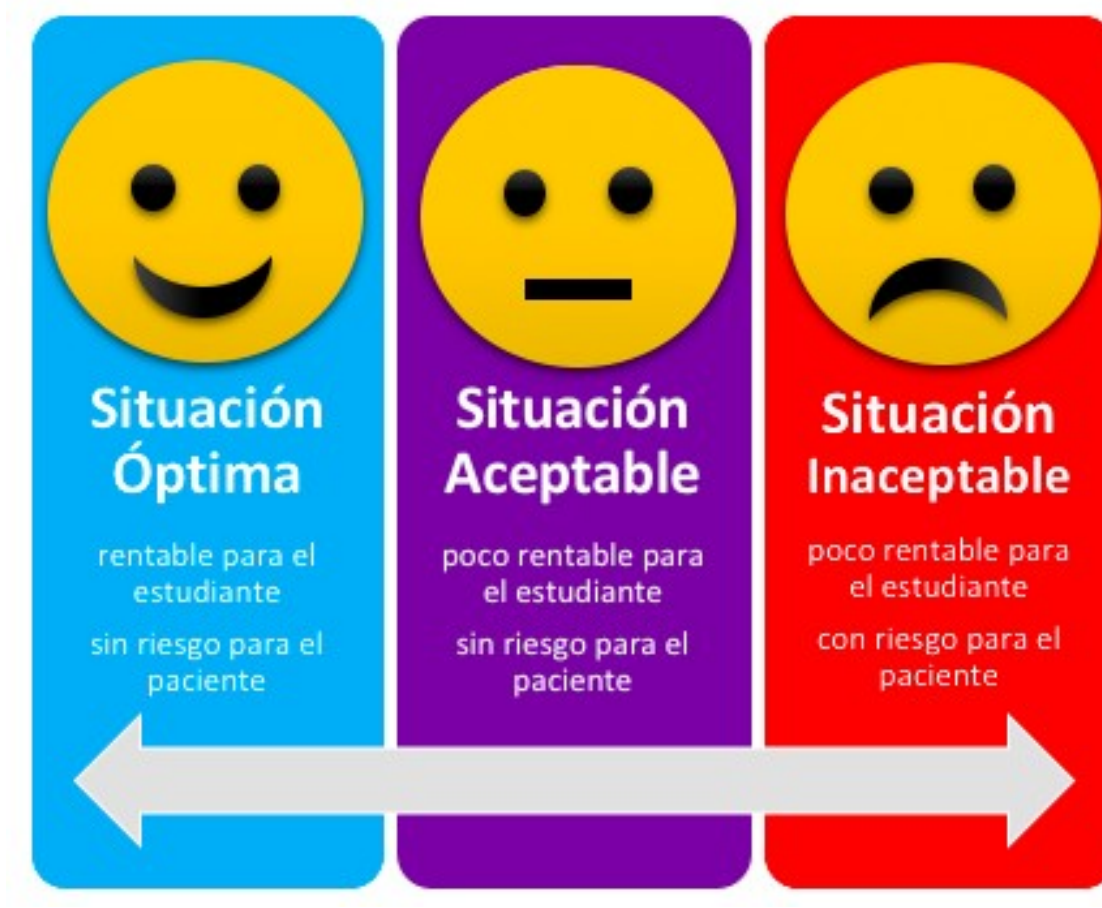

poco rentable para

el estudiante

sin riesgo para el paciente

poco rentable para

el estudiante

con riesgo para el paciente

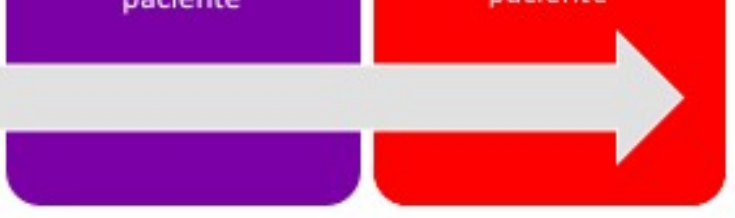

Figura 3. Propuesta de Esquema de Situaciones Clínicas en las que permitir la ejecución por parte del alumno. Elaboración propia.

Otro punto señalado por las pacientes que debemos tener en cuenta es el número de estudiantes, tanto desde el punto de vista del rendimiento que obtienen los alumnos, como de la sensación del paciente, que se puede sentir abrumado ante la presencia de muchos observadores.

"Mi primer parto fue presenciado por bastantes estudiantes. Resultó que una era conocida. Un poco de shock que me hubiera visto en esas... Por lo demás encantada, tendrán que aprender. Les animo y me aguanto si me hacen más daño".

"Obviamente hay situaciones en las que cuanta menos gente esté mirando más segura te sientes. Pero tienen que aprender y no es lo mismo tener a un estudiante que a seis mirando".

En aspectos quirúrgicos o tocúrgicos, la presencia de varios estudiantes minimiza su participación activa. Del mismo modo, en una consulta, la presencia de más de un alumno incita a una menor participación, preguntas al médico, etc. Por tanto, deberíamos intentar garantizar que haya un solo estudiante en cada proceso.

La mayoría de las pacientes, acorde a las respuestas del cuestionario, manifestaron mensajes muy positivos y en la mayoría de los casos se muestra que 
entienden la necesidad de la formación y les parece bien ser parte del proceso de formación del profesional médico. Algunas incluso recalcan la importancia del aprendizaje, no sólo de la enfermedad, sino del trato. Otro concepto clave y muy mencionado fue el entendimiento de que los estudiantes son los profesionales del futuro.

"Excelente, porque sirve para ayudar a formarlos como futuros profesionales".

"Necesaria. Serán nuestros médicos en el futuro".

"Soy profesora de Primaria y todo lo que sea beneficioso para el aprendizaje me parece estupendo".

"Fundamental para su formación".

"Que la gente aprenda con "fuego real" es positivo".

"Muy bueno, todos necesitamos aprender y qué mejor que viendo lo que ponen los libros en la práctica. Además, el médico siempre está supervisando todo y entre los dos pueden hacer un buen diagnóstico".

"Tienen que aprender y estar presentes en todo momento junto con los profesionales con más experiencia. Creo que es lo mejor para formarse".

"Me parece que es necesario que aprendan, son quienes nos cuidarán mañana".

"Es necesario y clave para que, además de formación académica, aprendan empatía con sus futuras pacientes".

"A mí me gusta porque creo que es la mejor manera de que aprendan, no sólo sobre medicina, sino sobre trato humano. Además, son los médicos del futuro".

"Me parece genial porque creo que para su formación es súper importante. Son los profesionales del futuro".

"Genial, tienen que aprender. Y además creo que de aprender así mucho más".

"Deben estar presentes, pues si no, no se pueden formar".

“Tienen que aprender y se aprende con prácticas. jAdelante!".

Para finalizar, creo que, de esta sencilla experiencia, se pueden extraer importantes conclusiones.

En primer lugar, considero importante que los alumnos sean conscientes, no por mera opinión o comentario, sino con datos reales, que las pacientes comprenden que tienen que estar ahí y que, de hecho, les gusta formar parte de su proceso de aprendizaje. Consideran la interacción con el paciente un elemento importante para su formación como los profesionales del futuro (como se puede observar en la Figura 4). Sin duda, este concepto les ayudará a afrontar su 
experiencia de una manera mucho más relajada y se permitirán a sí mismo adentrarse más en el proceso clínico.

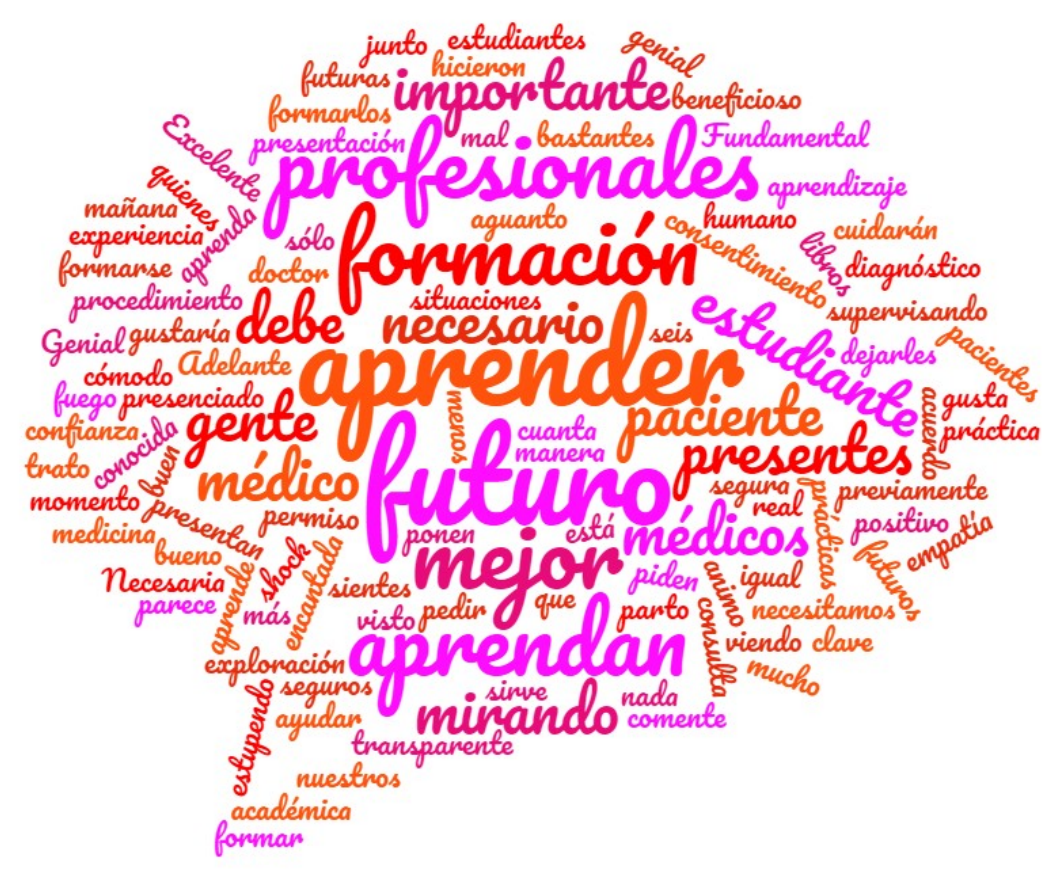

Figura 4. Nube de palabras formada con las opiniones escritas por las pacientes ante la pregunta abierta: "¿Qué os parece la presencia de estudiantes?". *Realizada usando la web wordclouds.com

En segundo lugar, basándonos en las opiniones y experiencias de estas pacientes, deberíamos establecer unas condiciones innegociables para optimizar la relación paciente-estudiante y lograr que se convierta en una experiencia inmersiva y con un rendimiento discente óptimo para el alumno, pero a la vez segura y confortable para el/la paciente. Estas serían:

- El médico debería explicarle a la paciente que va a haber un estudiante en el proceso clínico (consulta, pase de planta, cirugía, parto...) y pedir su consentimiento.

- El estudiante debería presentarse adecuadamente, con nombre y apellidos, así como su condición de estudiante y el curso académico al que pertenece. Del mismo modo que su tutor clínico, debe pedir permiso al paciente antes de explorar, tratar, entrevistar...

- Se debería mantener una relación de 1:1 entre alumno y médico, de manera que no coincida más de un estudiante con el mismo paciente.

- Antes de permitir que un estudiante ejecute una acción (prueba, procedimiento quirúrgico, exploración) hay que asegurarse de que tiene 
experiencia suficiente (ensayada con modelos de simulación) y que verdaderamente le aporta valor a su formación.

Financiación: No ha habido financiación.

Agradecimientos: A todas las pacientes y estudiantes que participaron con su valiosa opinión.

Declaración de conflicto of interés: El autor declara no tener ningún conflicto de intereses.

\section{Referencias}

1. Palés-Argullós J, Gomar-Sancho C. Repercusiones de la COVID-19 en la educación de los médicos: la formación de grado (II). FEM, 2020, 23 (4), 161-166. http:// doi.org/10.33588/fem.234.1076

2. Abo El-Nasr, Al-Badrawy. (2017). Role of Engineering Design in Enhancing ABET Outcomes of Engineering Programs at Taif University. IJAST, 6, 9-15. https://www.researchgate.net/publication/ 317267917 Role of Engineering Design in Enhancing ABET Outcomes of Engineering Programs at $\mathrm{T}$ aif_University

3. Berwanger J, Denti de Geroni G, Bonamigo EL. Estudiantes de medicina en la percepción de pacientes. Rev bioet. 2015, 23(3), 555-65. http://dx.doi.org/10.1590/1983-80422015233092

4. Sousa Muñoz RL, Ronconi DE, Ramalho CO, Andrade MR, Silva APF, Pereira GCB et al. Opinión de los pacientes sobre su participación en la enseñanza práctica de medicina en un hospital universitario de Brasil. Educ Med, 2011, 14(1), 35-37. http://scielo.isciii.es/pdf/edu/v14n1/original2.pdf

5. Shetty PA, Magazine R, Chogtu B. Patient outlook on bedside teaching in a medical school. Journal Talibah Iniv Med Sciencies, 2021,16(1),50-56. https://doi.org/10.1016/i.jtumed.2020.10.002

(C) 2021 Universidad de Murcia. Enviado para su publicación en acceso abierto bajo los términos y condiciones de la licencia Creative Commons Reconocimiento-NoComercial$\begin{array}{lllll}\text { Sin Obra } & \text { Derivada } & 4.0 \quad \text { España } & \text { (CC } & \text { BY-NC-ND) }\end{array}$ (http://creativecommons.org/licenses/by/4.0/). 